\title{
THE CHEMICAL COMPOSITION AND ORBITAL PARAMETERS OF BARIUM STARS
}

\author{
LAIMONS ZAČS \\ Radioastrophysical Observatory \\ Latvian Academy of Sciences \\ LV-1527 Riga, Latvia
}

\begin{abstract}
The observational relation between $s$-process abundance anomalies and orbital periods for barium stars is discussed and compared with mass-transfer simulations. Recent detailed abundance analyses of a large sample of single-lined long-period binaries provide evidence that all giants with white dwarf companions are likely to have abundance anomalies.
\end{abstract}

\section{Introduction}

In 1987 when as an undergraduate student I started my study of barium stars, it seemed to me that all was known about these peculiar objects, because abundance analyses confirmed the overabundance of carbon and $s$-process elements in their atmospheres (see Lambert 1985, and references therein), and their binarity (McClure et al. 1980; McClure 1983) and the white dwarf (WD) nature of the companion (Böhm-Vitense 1980; BöhmVitense et al. 1984) indicated that a transfer of heavy-element-rich matter from an AGB companion (now a WD) to the pre-barium star is a good explanation for the chemical peculiarities of the latter. Only some time later I saw that there are several critical questions that need additional observational tests.

\section{Is There a Difference between Variable and Non-Variable Radial-Velocity Examples among Barium Stars?}

The previous differentiation between classical (strong) and mild barium stars suggested by McClure et al. (1980) and Sneden et al. (1981) is not clear physically. Furthermore, McClure and Woodsworth (1990) found several barium stars that have shown no radial velocity (RV) variations. It is clearly of interest to know if these two groups of barium stars also have 


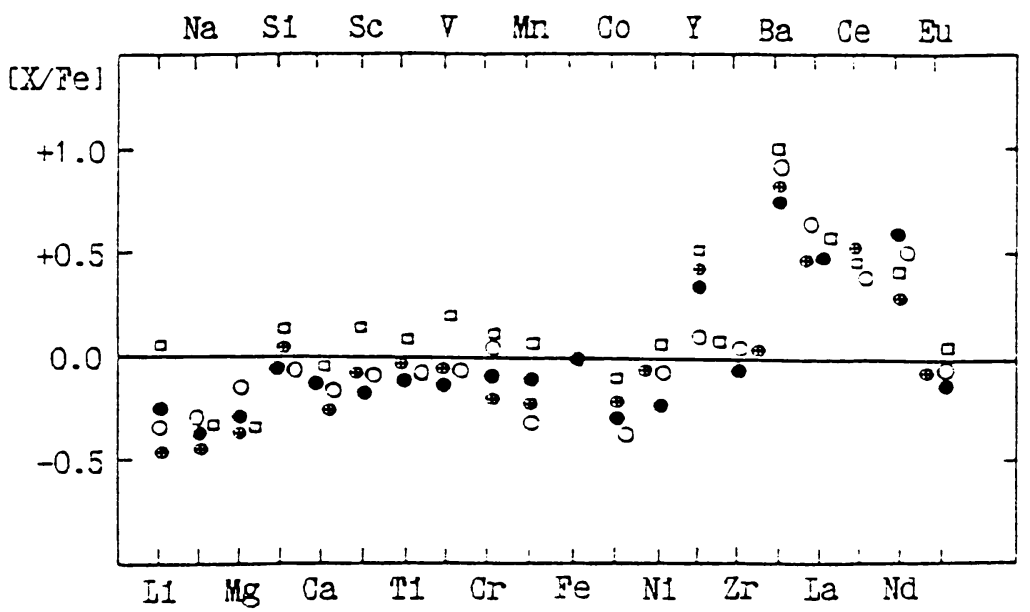

Figure 1. Differential abundance comparisons for two barium stars with variable radial velocity (crossed circles: HD 205011; squares: HD 131670) and two non-variable examples (filled circles: HD 65854; open circles: HD 104979).

different physical properties (Začs 1994). A comparison of the abundance patterns for four radial velocity variable and nonvariable examples with similar peculiarity levels is shown in Figure 1. There is no significant difference in the abundances of these stars. The absolute magnitudes and atmospheric parameters are similar as well. Thus the quantitative analyses show that both groups of barium stars apparently belong to a single family of peculiar giants. This means that the mechanism responsible for the abundance peculiarities is probably common to both.

Two RV-nonvariable mild barium stars from Jorissen's list (Jorissen 1994) have been analyzed recently (Začs et al. 1997) using high S/N CCD spectra covering the wide spectral region $5000-7200 \AA$. A significant enhancement of $s$-process elements was found for these two stars: HD 130255 (G4 IV, Ba 1.0, [Ba II/Fe II] = +0.79 dex) and HD 119185 (K0 III, Ba 1.0, $[\mathrm{BaII} / \mathrm{Fe} \mathrm{II}]=+0.35 \mathrm{dex})$. These observations confirm the conclusion that RV-nonvariable barium stars have either very long orbital periods or highly inclined orbital planes. Certainly, it is possible that some normal red giants have been classified by mistake as mild barium stars.

\section{The Relation between Level of Chemical Anomalies and Orbital Period}

If a transfer of heavy-element-rich matter from an AGB companion to the pre-barium star is the explanation for the chemical peculiarities of barium stars, there should be a correlation between the level of chemical anomalies 



Figure 2. Comparison between the wind accretion model prediction (solid line) and the observational data (points) for different sets of parameters (Boffin \& Začs 1994).

and the orbital period (Boffin \& Jorissen 1988; Han et al. 1995). This prediction has been tested on the basis of mean abundance data of high homogeneity for a large sample of barium stars with known orbital elements (Začs 1994). To minimize observational errors, the overabundances of $s$ process elements in barium stars are characterized by an index: $[s]=([\mathrm{Y} / \mathrm{Fe}]$ $+[\mathrm{Ba} / \mathrm{Fe}]+[\mathrm{La} / \mathrm{Fe}]+[\mathrm{Ce} / \mathrm{Fe}]+[\mathrm{Nd} / \mathrm{Fe}]) / 5$. The relation between the orbital period and the observed overabundance of $s$-process elements is confirmed, although it is clear that a one-to-one relation does not exist: for a given period, various overabundances may occur.

\section{Mass Transfer Mechanism}

The first quantitative confrontation of observational data for a homogeneous sample of barium stars with theoretical predictions for the wind accretion scenario (Boffin \& Jorissen 1988) confirmed that the global trend of the relation (abundance peculiarity-orbital period) can be obtained with the wind accretion model (Boffin \& Začs 1994). Better agreement with the observations is obtained either by increasing the wind velocity or by de- 


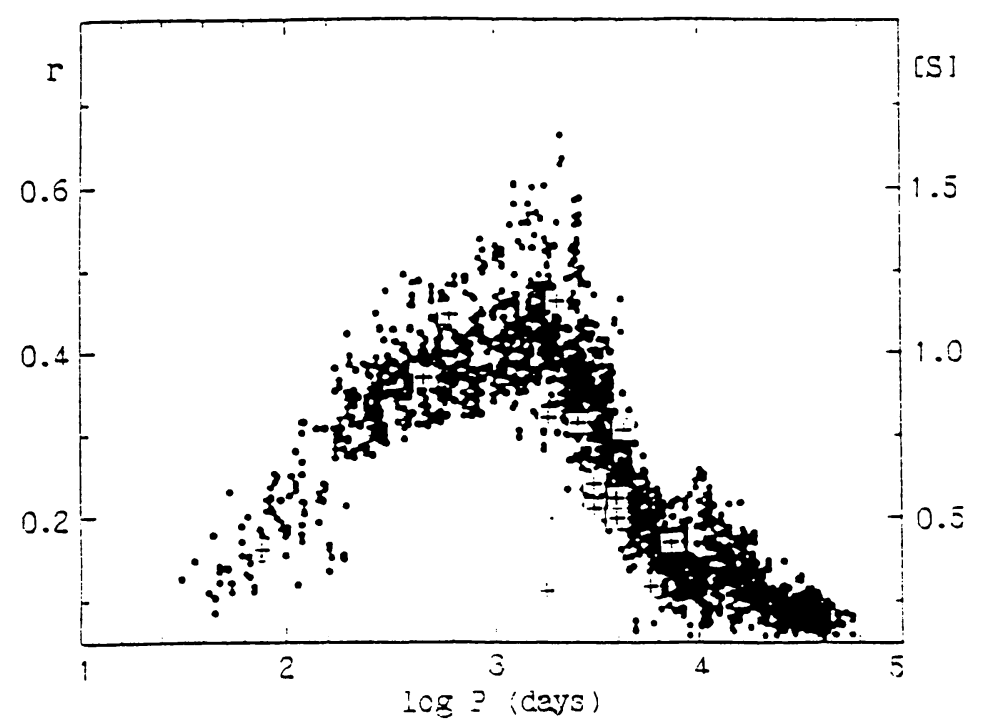

Figure 3. Comparison between the combined model predictions (points) and the observational data (plus signs). $P$ is the orbital period, and $r$ is the pollution factor (Han et al. 1995).

creasing the initial overabundance of $s$-process elements in the atmosphere of the AGB star (Figure 2). Recent smoothed particle hydrodynamics simulations (Theuns, Boffin \& Jorissen 1996) have confirmed the efficiency of wind accretion to account for the chemical peculiarities for orbital periods up to about 90 years; however, this scenario faces difficulties for systems with short orbital periods. The confrontation of observations (Začs 1994) with simulations for the combined mass transfer scenario which includes wind accretion, wind exposure, stable Roche lobe overflow, and commonenvelope ejection (Han et al. 1995) is shown in Figure 3. There is excellent agreement for both long and short period systems.

The confrontation of observations with mass-transfer simulations provides strong evidence that long-period $(P>3$ years) barium stars are mainly the result of wind accretion (and probably wind exposure) from an AGB companion. Roche lobe overflow and common-envelope ejection are probably the most important evolutionary channels for short period barium stars, although additional observations are required for more secure conclusions.

\section{Barium-Star-Like Long-Period Single-Lined Binaries}

Many spectroscopic long-period binaries with a red giant primary and an unseen secondary (probably a WD in some cases) whose orbits have similar 
characteristics to those of barium stars have in all probability solar abundances of the heavy elements, including four normal giants $\left(\xi^{1}\right.$ Cet, DR Dra, HD 21120, HD 81817) with directly observed WD companions (Jorissen \& Boffin 1992; Boffin et al. 1993; Fekel et al. 1993). The existence of such giants led the authors to conclude that the existence of a WD companion in a barium-star-like system is not sufficient to produce a barium star. However, small abundance anomalies are difficult to detect, especially on the basis of a spectrum in a narrow spectral region. For example, Pilachowski (1977) and Začs (1994) suggested that $\xi^{1}$ Cet (HD 13611) has a mild $s$ process enhancement. An important step toward a better understanding of the phenomenon of barium stars is a detailed abundance analysis of spectroscopic binaries with barium-star-like orbital elements (Začs et al. 1997), especially normal giants with directly observed WD companions.

\subsection{ARE THERE NORMAL GIANTS WITH WHITE DWARF COMPANIONS?}

Unfortunately, the answer to this question depends for the time being on the accuracy required in the definition of "normal". Certainly, there are at least some "normal giants" with WD companions having atmospheric anomalies less than 0.4 dex (see Jorissen \& Boffin 1992). However, only two such giants have been analyzed in detail using high resolution spectra in a wide spectral region. These analyses provide strong evidence that the atmospheres of $\xi^{1}$ Cet and DR Dra (Začs et al. 1997) are slightly peculiar! The question concerning the peculiarity of other candidates for normal giants remains unanswered, because $s$-process anomalies at the level of 0.3 dex can be confirmed only on the basis of spectra over a wide spectral region, ensuring high accuracy in the atmospheric parameters and abundance pattern obtained. A recent abundance analysis of long-period singlelined binaries with orbital elements similar to barium stars (Začs et al. $1997)$ indicated that all giants in the (eccentricity $(e), \log P$ ) plane (Figure 4 ) in the area occupied by typical barium stars have slight (0.2-0.3 dex) abundance anomalies. Thus it seems likely that all giants with WD companions in wide systems have $s$-process peculiarities. The level of peculiarity probably depends on the efficiency of the $s$-process and mass transfer in each specific binary system.

\subsection{CAN THE PRESENCE OF A COMPANION INFLUENCE THE INTERNAL STRUCTURE OF THE BARIUM STAR?}

It has sometimes been suggested that the phenomenon of barium stars may be connected to an unseen companion indirectly - not via mass transfer to a pre-barium star. In principle, a companion may have affected the pre- 


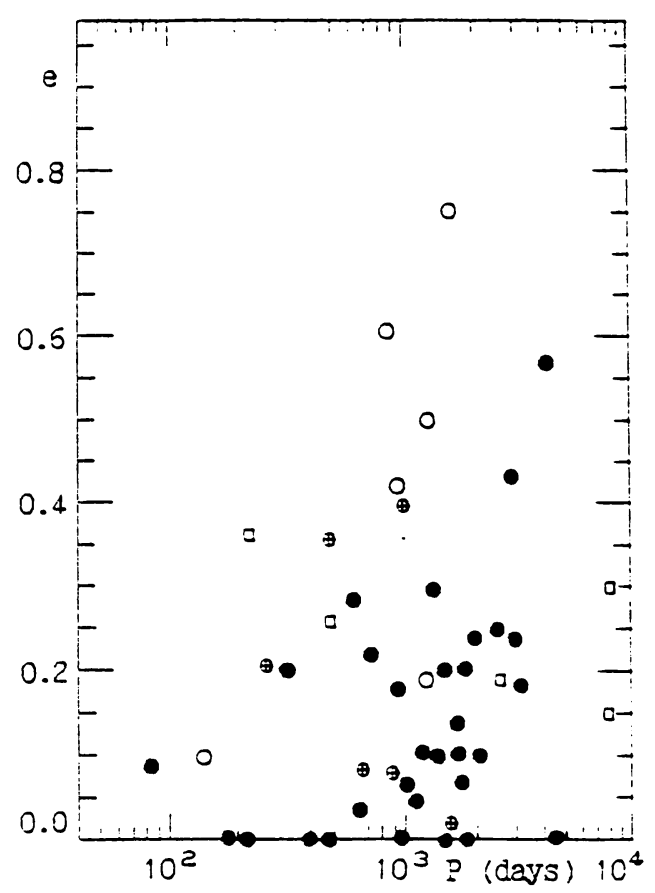

Figure 4. The $(e, \log P)$ diagram for single-lined spectroscopic binaries with barium-star-like orbital elements. Filled circles: barium stars from Jorissen \& Boffin (1992); open circles: giants showing normal barium abundance; crossed circles: giants showing mild barium enhancement; squares: dwarfs.

barium star's interior in some way which causes an extra degree of mixing (McClure 1984). This assumption has been tested for long-period binaries (Začs et al. 1997) with characteristics similar to barium stars but containing a main-sequence companion. The analysis indicates that a main-sequence companion does not have a significant influence on the internal structure (chemical composition) of the primary star.

\subsection{WHAT ARE THE SUFFICIENT CONDITIONS FOR THE FORMATION OF ABUNDANCE PECULIARITIES?}

In recent years there has been a tendency to consider that the presence of a WD companion in long-period systems is not sufficient for the barium star phenomenon, and additional parameters have been sought to explain the absence of abundance anomalies. Metallicity in particular has been considered as one such extra parameter. However, we need to explain why there exist a large number of barium giants such as $\mathrm{HD} 121447([\mathrm{Fe} / \mathrm{H}]=$ $+0.05,[\mathrm{Ba} / \mathrm{Fe}]=+0.57), \mathrm{HD} 100503([\mathrm{Fe} / \mathrm{H}]=+0.05,[\mathrm{Ba} / \mathrm{Fe}]=+0.56)$, and $\mathrm{CPD}-64^{\circ} 4333([\mathrm{Fe} / \mathrm{H}]=+0.05,[\mathrm{Ba} / \mathrm{Fe}]=+0.62)$ (see Smith 1984). 
In my opinion, if one or more barium stars were found to have a solar metallicity, this would mean that it is not necessary for the pre-barium system to be metal-deficient to produce the peculiarity. The observational fact that a relation exists between $[\mathrm{Ba} / \mathrm{Fe}]$ and $[\mathrm{Fe} / \mathrm{H}]$ (Kovacs 1985) i.e. more extreme barium stars are more iron-deficient (older) than the stars with small overabundances of barium - does not mean that a low metallicity is one of the parameters which determines whether a normal giant becomes peculiar. We should take into account that there is a relation between abundance peculiarity and orbital period for barium stars. For example, the two stars HR 774 and HD 178717 have a difference in orbital periods (848 days) corresponding to a difference of $\sim 0.3$ dex in $[s]$ (see Fig. 3), which is in agreement with the $([\mathrm{Ba} / \mathrm{Fe}], \mathrm{Fe} / \mathrm{H}])$ relation (see Fig. 10 in Kovacs 1985). It is clearly of interest to investigate the influence of binarity (strong barium stars have larger radial velocity amplitudes than mild barium stars) on the space velocity dispersion (strong barium stars have a larger velocity dispersion, see $\mathrm{Lu} \mathrm{1991).} \mathrm{In} \mathrm{any} \mathrm{case,} \mathrm{abundance}$ studies (Začs et al. 1997) give strong evidence that the existence of a white dwarf companion in binary systems with barium-star-like characteristics is not sufficient to produce a strong barium star, but it is perhaps premature to conclude that the existence of a WD in a barium-star-like system is not sufficient to create abundance peculiarities.

\section{Future Developments}

First, it will be very important to check whether the giants with directly confirmed WD companions (for example, HD 21120 and HD 81817) really do not have any $s$-process anomalies. Since WD companions have been confirmed only for some barium stars, investigations of "normal" long period giants where the companion is suspected to be a WD $(e<0.1, P>1000$ days; see Jorissen \& Boffin 1992) would be very useful. For further improvement, high $\mathrm{S} / \mathrm{N}$ spectra over wide spectral regions are necessary to test in detail the element-by-element composition.

Second, it is important to look on the barium stars as a phenomenon caused by polluted mass transfer in binary systems, independent of the present evolutionary status of the primary. In this connection we should mention the recent detailed abundance analysis of AG Dra (Cunha et al. 2000) and the Monte Carlo simulations by Han et al. (1995) which try to explain a large variety of peculiar stars including cataclysmic variables and symbiotics on the basis of the mass-transfer mechanism. Detailed abundance analyses of peculiar stars such as HR 1105 (S3.5/2, $P=596.21$ days, $e=0.09, f(m)=0.0375$ ) and $\tau \mathrm{UMa}$ (Am, $P=1062.4$ days, $e=0.48$, $f(m)=0.0044)$ will enrich our knowledge of the barium star phenomenon. 
If it has sometimes been suggested that the phenomenon of barium stars may possibly be the result of a combined process of mass transfer and stellar evolution, so too the phenomenon of long period metallic-line stars may be the result of mass transfer and diffusion in the atmospheres of A stars. On the other hand, examination of the correlation between abundance anomalies and orbital periods for barium-related stars $(\mathrm{CH}$ stars, subgiant $\mathrm{CH}$ stars) may allow us to find the general principle of the barium star phenomenon.

Third, an examination of s-process parameters and the neutron source is needed for the families of barium-related stars. Although many advances have been made in recent years, further progress on a large scale may depend on the confrontation of the averaged $s$-process abundance patterns for homogeneous samples of peculiar red giants with $s$-process simulations.

I am grateful to my teachers and collaborators V. E. Panchuk, V. G. Klochkova, I. F. Bikmaev, H. M. J. Boffin, and F. A. Musaev. My work is partially supported by the ESO C\&EE Committee (through grants B-01-012 and E-06-002). I thank the IAU and the symposium SOC (chaired by R. Wing) for travel support.

\section{References}

Boffin, H. M. J., Cerf, N. \& Paulus, G. 1993, $A \& A, 271,125$

Boffin, H. M. J. \& Jorissen, A. 1988, $A \& A, 205,155$

Boffin, H. M. J. \& Začs, L. 1994, $A \& A$, 291, 811

Böhm-Vitense, E. 1980, ApJ, 239, L79

Böhm-Vitense, E., Nemec, J. \& Proffitt, C. 1984, ApJ, 278, 726

Cunha, K., Smith, V. V. \& Jorissen. A. 2000, in IAU Symposium 177: The Carbon Star Phenomenon, ed. R. F. Wing (Kluwer), p. 103

Fekel, F. C., Henry, G. W., Busby, .M. R. \& Eitter, J. J. 1993, AJ, 106, 2370

Han, Z., Eggleton, P. P., Podsiadlowski, P. \& Tout, C. A. 1995, MNRAS, 277, 1443

Jorissen, A. 1994, private communication

Jorissen, A. \& Boffin, H. M. J. 1992, in Binaries as Tracers of Stellar Formation, ed. A. Duquennoy and M. Mayor (Cambridge Univ. Press), p. 110

Kovacs, N. 1985, $A \& A, 150,232$

Lambert, D. 1985, in Cool stars with Excesses of Heavy Elements, ed. M. Jaschek and P. C. Keenan (Reidel), p. 191

Lu, P. K. 1991, $A J, 101,2229$

McClure, R. D. $1983, A p J, 268,264$

McClure, R. D. 1984, PASP, 96, 117

McClure, R. D., Fletcher, J. M. \& Nemec, J. M. 1980, $A p J, 238$, L35

McClure, R. D. \& Woodsworth, A. W. 1990. ApJ, 352, 709

Pilachowski, C. A. $1977, A \& A, 54,465$

Smith, V. V. $1984, A \& A, 132,326$

Sneden, C., Lambert, D. L. \& Pilachowski, C. A. 1981, ApJ, 247, 1052

Theuns, T., Boffin, H. M. J. \& Jorissen, A. 1996, MNRAS, 280, 1264

Začs, L. 1994, $A \& A, 283,937$

Začs, L., Musaev, F.A., Bikmaev, I. F. \& Alksnis, O. 1997, A\&A Supp., 122, 31 